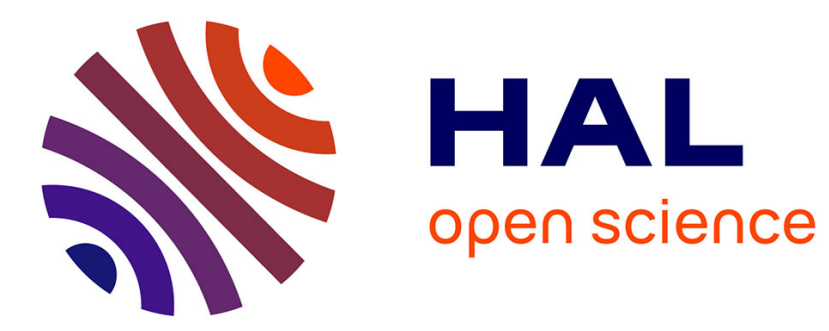

\title{
Collateral constraints and the amplification-persistence trade-off
}

\author{
Patrick-Antoine Pintus
}

\section{To cite this version:}

Patrick-Antoine Pintus. Collateral constraints and the amplification-persistence trade-off. 2009. halshs-00439243

\section{HAL Id: halshs-00439243 \\ https://shs.hal.science/halshs-00439243}

Preprint submitted on 7 Dec 2009

HAL is a multi-disciplinary open access archive for the deposit and dissemination of scientific research documents, whether they are published or not. The documents may come from teaching and research institutions in France or abroad, or from public or private research centers.
L'archive ouverte pluridisciplinaire HAL, est destinée au dépôt et à la diffusion de documents scientifiques de niveau recherche, publiés ou non, émanant des établissements d'enseignement et de recherche français ou étrangers, des laboratoires publics ou privés. 

OFF

\section{Patrick PINTUS}

September 2009

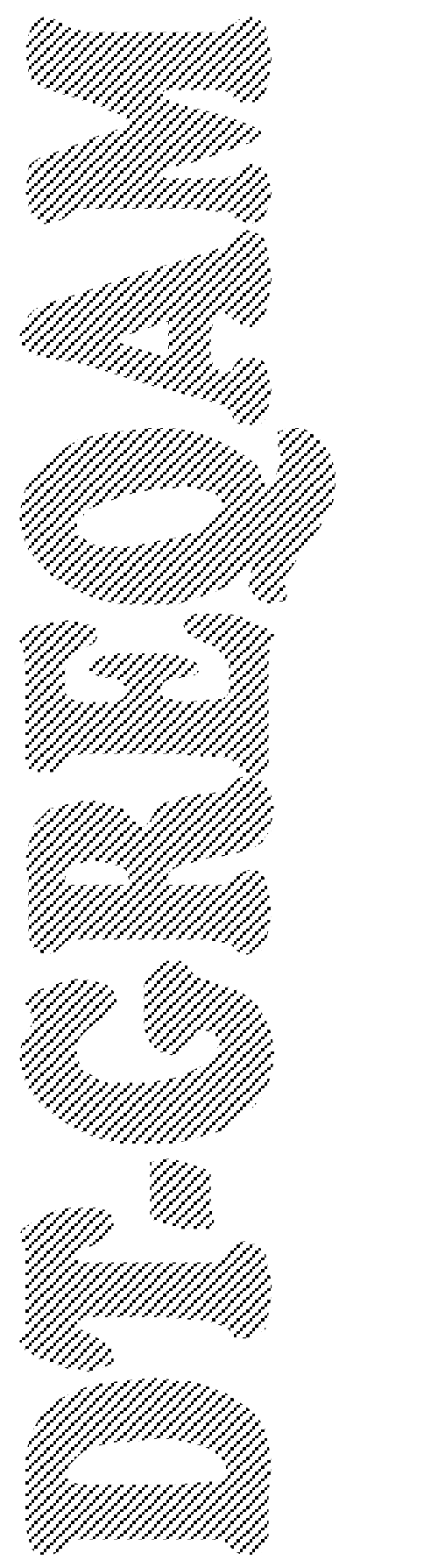




\title{
Collateral Constraints and the Amplification-Persistence Trade-off*
}

\author{
Patrick A. Pintus ${ }^{\dagger}$ \\ Université de la Méditerranée and GREQAM-IDEP
}

September 17, 2009

\begin{abstract}
Kiyotaki and Moore (1997) have stressed that an amplification-persistence trade-off arises when collateral constraints on borrowing interact with lumpy investment. In this paper, I confirm by way of example that collateral constraints are not by themselves responsible for such a deceptive trade-off. More precisely, I show in a standard general-equilibrium two-agent model that the amplification and persistence of the impact of temporary shocks go hand in hand. Unlike Kiyotaki-Moore's, the economy features concave utility and production functions, an endogenous interest rate and neo-classical input accumulation.
\end{abstract}

Keywords: collateral constraints, amplification and persistence of aggregate shocks.

Journal of Economic Literature Classification Numbers: D92, E32, E44.

* The author wishes to thank Marty Eichenbaum, David Levine, Jean-Charles Rochet, Yi Wen, and seminar participants at various places for useful comments and suggestions on previous drafts. This paper was revised while the author was visiting the Research Division of the Federal Reserve Bank of St Louis, whose hospitality and financial support are gratefully acknowledged. In particular, this version draws on some insights generated by a related joint project with Yi Wen. The previous version of this paper was titled "Credit Market Frictions and the Amplification-Persistence Trade-off". This work was supported by French National Research Agency Grant (ANR-08-BLAN0245-01).

$\dagger$ E-mail: pintus@univmed.fr. 


\section{Introduction}

In their seminal contribution, Kiyotaki and Moore [7] (KM henceforth) have shown that credit cycles occur when collateral constraints interact with lumpy investment. In addition, they have also emphasized that such an interplay originates an amplificationpersistence trade-off. The reason why this trade-off occurs is intuitively simple: collateralized borrowing amplifies shocks only if the proportion of credit-constrained firms is large enough, that is, only if the probability of investment opportunity is large enough. Persistence, on the contrary, requires the fraction of non-investing firms to be large enough. Not surprisingly, this is tantamount to the effect of nonadjusting firms in sticky-price models, in which more persistence arises if the fraction of inertial firms in the economy is larger.

Such a trade-off arguably casts some doubts on the quantitative importance of collateral constraints. For example, this trade-off dictates that credit constraints amplify shocks to total factor productivity (TFP henceforth) for some parameter values, but that persistence is typically small for such parameter constellations. In other words, the possibly large effects of shocks at impact are bound to be shortlived. Conversely, large persistence of the impact of shocks is associated with small amplification. KM [6] and [7] emphasize that their assumption of lumpy investment is essential to the occurrence of such a rather disappointing trade-off. Therefore, an open question is whether substituting neo-classical input accumulation for lumpy investment in an economy with collateral constraints rules out the trade-off. Although 
KM touch upon this issue, they do so in a setting that relies on some specific and questionable assumptions. For example, KM assume that the utility and production functions are linear and that the interest rate is exogenous. As a consequence, one is left wondering what is the exact role played by these ingredients, as opposed to the collateral constraints, in the amplification-persistence trade-off.

The main purpose of this paper is to shed some light on the answer to this question, by way of a simple example. I study a general-equilibrium version of a two-agent economy that incorporates collateral constraints and neo-classical capital accumulation. The interest of such a framework is that it is as close as possible to a benchmark business-cycle model, while retaining KM's collateral constraints. Therefore, amplification and propagation can be entirely attributed to credit market frictions $\grave{a} l a$ KM. The most important lesson one draws from examining the impulse-response functions of such a model is that the trade-off originated in KM's model does not survive: amplification and persistence of the impact of shocks turn out to go hand in hand. In particular, increasing the relative risk aversion on the borrower's side not only magnifies the amplification of a temporary, unexpected and serially uncorrelated TFP shock in the period following impact. This also enlarges the persistence of the impact of the TFP shock. The intuition for why the trade-off disappears, as developed in Section 3, is that the larger risk aversion and consumption smoothing, the more pronounced amplification and persistence.

This paper is, of course, not the first to discuss how shocks propagate in economies with collateral constraints. Among others, Kocherlakota [8] works out an open- 
economy model which exhibits a similar trade-off but relies on an exogenous interest rate, just as KM's. Cordoba and Ripoll [3] study a model that shares many similarities with the setting considered in this paper. However, these authors introduce reproducible land but not reproducible capital, so that their model is less comparable to KM's than the setting considered in this paper.

In Section 2, the amplification-persistence trade-off is restated and discussed. The main result of this paper is developed in Section 3, where I study an extended version of KM's model with concave preferences and technology. In Section 4, I state some concluding remarks and conjectures while the final appendix collects some proofs.

\section{The Amplification-Persistence Trade-off in the Kiyotaki-Moore Economy: A Restatement}

This section is devoted to restating and discussing the amplification-persistence trade-off that arises in the full model with credit-constrained investment by heterogeneous firms of KM [7, Section III]. The economy is composed of borrowers-farmers that are supposed to be less patient than lenders-gatherers. Among the borrowers, a fraction $1 \geq \pi \geq 0$ receives an investment opportunity and is credit-constrained while the remaining fraction $1-\pi$ cannot invest and therefore scales down its scale of operation because capital depreciates. To save space, I simply recall the following definition stating the three main equations that drive the dynamics of the economy. 
Details on the derivation are found in KM [7, pp. 215-233].

\section{Definition 2.1 (Competitive Equilibrium)}

An intertemporal competitive equilibrium with perfect foresight is a sequence of positive land stock, debt stock and land price $\left(L_{t}, B_{t}, Q_{t}\right)$, for all $t \geq 0$, such that, given some initial values $L_{0}, B_{0}>0$ :

$$
\left\{\begin{aligned}
L_{t+1} & =(1-\pi) \lambda L_{t}+\pi\left[\left(a+Q_{t+1}+\lambda \phi\right) L_{t}-R B_{t}\right] /\left[\phi+u\left(L_{t+1}\right)\right] \\
B_{t+1} & =R B_{t}+Q_{t+1}\left(L_{t+1}-L_{t}\right)+\phi\left(L_{t+1}-\lambda L_{t}\right)-a L_{t} \\
Q_{t+1} & =R\left[Q_{t}-u\left(L_{t}\right)\right]
\end{aligned}\right.
$$

where $u(L) \equiv G^{\prime}[(\bar{L}-L) / m] / R$ denotes the user's cost of holding land.

In eqs. (1) (which correspond to KM's [7] eqs. (23)-(24) and (12), respectively), $\bar{L}$ is the (constant) stock of land supply to be shared among farmers and gatherers, $L$ is the stock of farmers' land, $B$ is the stock of farmers' debt, $Q$ is the land price (per unit of fruit, the numéraire), $R>1$ is the constant gross interest rate, $m>0$ is the proportion of gatherers, $1 \geq 1-\lambda \geq 0$ is the rate of tree depreciation. The remaining parameters are $a \geq 0$, the productivity level of farmers' technology, and $\phi \geq 0$, the unit cost of investing in trees.

The last equation in (1) expresses equilibrium on the land market, as it equates the marginal product of land $u\left(L_{t}\right)$ to the user's cost of holding land $Q_{t}-Q_{t+1} / R$. The second equality in (1) is the borrowers' budget constraint: tomorrow's debt stock 
results from the excess over tradable output of debt repayment from the previous period, land investment, and investment in trees. Finally, the first equation in (1) is the law of capital accumulation: tomorrow's land stock comes from the addition of the undepreciated stock from the fraction $1-\pi$ of non-investing farmers and of the land stock provided by the fraction $\pi$ of investing farmers.

$\mathrm{KM}\left[7\right.$, p. 233] show that eqs. (1) have a unique interior steady state $\left(L^{*}, B^{*}, Q^{*}\right)$ such that the collateral constraint is binding for investing farmers (although not for non-investing farmers), provided that the following restrictions on the parameters are imposed:

\section{Assumption 2.1}

The following restrictions on parameters are assumed:

$$
\begin{gathered}
a>\phi(1-\lambda), \pi>1-1 / R, \\
c>(1 / \beta-1)(a+\phi)[1-\beta R \lambda(1-\pi)] /[\beta R(\lambda \pi+(1-\lambda)(1-R+\pi R))],
\end{gathered}
$$

where $c>0$ determines bruised output $c K$. In (2)-(3), the inequalities respectively ensure that there is enough tradable output to replace the depreciated trees, that the probability of investment is not too small, and that the return from farming is large enough that all tradable production is used for investment. In addition, exploding bubbles are ruled out by the usual "no-Ponzi game condition", that is $\lim _{s \rightarrow \infty} E_{t}\left(R^{-s} Q_{t+s}\right)=0$. 
The main goal of this section is to restate and discuss the amplification-persistence trade-off that emerges from the model. The main parameter of interest is $\pi$, the fraction of credit-constrained farmers. As acknowledged by KM [6, Section 4], heterogeneity is an essential element of the model to get credit cycles, whereas trees are not. In other words, credit cycles require $\pi<1$ while they do occur even if $\phi=0$ (so that only investment in land, not in trees, is allowed). As the analytics is quite cumbersome when both $\pi<1$ and $\phi>0$ and because trees are not essential for KM's main result while heterogeneity certainly is, I state the next proposition under the assumption that $\pi<1$ and $\phi=0$. However, KM [7, pp. 236-8] discuss some numerical examples that confirm the robustness of the amplification-persistence trade-off when $\phi>0$.

A first step is to linearize eqs. (1) at $\left(L^{*}, B^{*}, Q^{*}\right)$ (see the appendix in KM [6] for some details of the derivation), which delivers that $R>1$ is an (unstable) eigenvalue while the remaining eigenvalues are the roots of the second-order polynomial $p(x)=x^{2}-T x+D$, with:

$$
\begin{aligned}
D & =\eta \lambda R(1-\pi) /(\eta+1-\lambda+\lambda \pi), \\
T & =D+[\eta+R(1-\pi)(1-\lambda)] /(\eta+1-\lambda+\lambda \pi),
\end{aligned}
$$

where $\eta>0$ is the elasticity of the residual supply of land to the farmers with respect to the user's cost, evaluated at steady state (see KM [7, p. 225]). Notice that if the period is interpreted as being short (say, a quarter), then $R$ is close to one and, therefore, the unstable eigenvalue is close to unit root. Therefore, the steady state is a saddle if the two remaining eigenvalues are stable, as there are two predetermined 
variables in eqs. (1), namely the stocks $L$ and $B$, while the land price $Q$ is a jump variable. As I now show, the two roots of the polynomial in (4) are stable under the maintained assumptions.

\section{Proposition 2.1 (Saddle-Point Stability of the Steady State)}

Under Assumption 2.1 and $\phi=0$, the steady state $\left(L^{*}, B^{*}, Q^{*}\right)$ of eqs. (1) is locally a saddle (hence determinate).

Proof: See Appendix A.

In a previous version of this paper, Pintus [10], I show that when the conditions of the previous proposition are relaxed, then the steady state may undergo a Hopf bifurcation when $\pi$ goes through a critical value that is typically small. As a consequence, endogenous cycles may surround the steady state. To save space, the interested reader is referred to the earlier version for details on local bifurcations occurring in the KM model.

I am now ready to restate the amplification-persistence trade-off. From the details contained in the proof of Proposition 2.1, one gets that the two stable roots of $p(x)$ are complex provided that $\pi$ is not too close to one. As already shown by KM $[7$, p. 234], heterogeneity leads to a linearized model that is $A R(2)$ and it is necessary for credit cycles to occur. In the sequel, I focus on the configuration where $p(x)$ has complex roots. Persistence is defined as the modulus of the complex eigenvalues, that is, $\sqrt{D}$. Alternatively, following KM, persistence is inversely measured by the decay 
rate $\mu \equiv 1-\sqrt{D}$. In Proposition 2.1 , the steady state is a saddle that possesses one unstable eigenvalue (that is, $R>1$ ) and two eigenvalues lying inside the unit circle. Therefore, the requirement that $\lim _{s \rightarrow \infty} E_{t}\left(R^{-s} Q_{t+s}\right)=0$ for all $t \geq 1$ ruling out exploding bubbles implies that the dynamics are restricted to the stable manifold of the saddle, where they exhibit damped oscillations that decay at rate $\mu$. Then persistence is "maximal" when complex unit roots occur, that is, when $D=1$ or, equivalently, $\mu=0$. On the other hand, amplification is measured by the deviation from steady state occurring after a one-period productivity shock hits the economy, while it is at steady state. For example, let $\Delta$ denote the (small) initial productivity shock, in percentage terms, and let $\hat{Y} \equiv\left(Y-Y^{*}\right) / Y^{*}$ define the next-period deviation of output from steady state, where $Y^{*}$ is steady state output. Then the output amplification is $\hat{Y} / \Delta$. Analogous definitions hold for the amplification of land price, $\hat{Q}$, and of land stock $\hat{L}$.

\section{Theorem 2.1 (The Amplification-Persistence Trade-off)}

Under the assumptions of Proposition 2.1, assume that the polynomial in eqs. (4) has complex roots and that the depreciation rate of trees is close enough to zero (that is, $\lambda$ is close enough to one). Then the dynamics of eqs. (1) near the steady state are such that both the decay rate and the amplification of a temporary, unexpected productivity shock on capital and output increase when $\pi$ goes up.

In other words, there exists an amplification-persistence trade-off. 
Proof: See Appendix B.

The intuitive discussion of the main mechanisms at work provided by KM emphasizes that the trade-off is linked to the assumption of lumpy investment. This can be seen by supposing a positive TFP shock and recalling that only a fraction $\pi$ of farmers faces an investment opportunity each period and can react to such a disturbance. As a consequence, the higher $\pi$, the larger the amplification of the impact of the temporary shock: the larger $\pi$, the larger the increase of demand for land from farmers that is accommodated by gatherers, the higher the increase in the land price, and the more relaxed the credit constraint is. Hence, the larger the increases in land reallocation, investment and output. On the other hand, when the fraction of investing firms is large, then the endogenous persistence of the shock impact turns out to be low, because almost all farmers increase their scale of operation through borrowing and investment while only a small fraction leaves their stock of cultivated land almost constant (if, plausibly, the rate of tree depreciation is small). ${ }^{1}$ In summary, the key point behind this trade-off is that persistence requires the fraction of credit-constrained firms to be small enough, so that a low fraction of farmers invest in each period, while high amplification relies on just the opposite condition, as it occurs only when there are many credit-constrained firms. This is similar to the effect of non-adjusting firms in sticky-price models, in which persistence is larger if the fraction of inertial firms in the economy is large enough.

\footnotetext{
${ }^{1}$ More technically, direct examination of eqs. (1) reveals that persistence in the dynamics of the farmers' stock of land is large when both $\lambda$ is close to one and $\pi$ is close to zero.
} 
Theorem 2.1 focuses on investment heterogeneity, through the parameter $\pi$, because this feature is key to obtain credit cycles, as shown by KM. However, varying other parameters also reveals the amplification-persistence trade-off. In particular, it is not difficult to show that persistence is an increasing function - while amplification is a decreasing function - of $\eta, \phi$ and $R$. Here again, the trade-off manifests itself. For example, the intuition shedding light on the effects of $\eta$ is as follows. Remember that $\eta$ is the elasticity of the residual supply of land to the farmers with respect to the user's cost. When a positive TFP shock hits the economy, the user's cost of holding land increases, which partly attenuates the increase in investment following the shock. Therefore with a large $\eta$ is associated a small amplification. On the other hand, large $\eta$ 's imply large persistence. It is useful to think about the limiting case in which $\eta$ is infinite: in this configuration, the land price does not move after the shock and therefore there is less amplification, but persistence is complete because land reallocation from gatherers to farmers becomes permanent.

\section{Is the Amplification-Persistence Trade-off Ro- bust?}

The answer provided by this section is 'No'. Given the auxiliary assumptions made by KM, one is left wondering to what extent the deceptive amplification-persistence trade-off is due to collateral constraints. In particular, credit cycles are shown by KM 
to occur under the assumptions both that investment is lumpy and that the utility and production functions are linear, implying that the interest rate is exogenous. What is the exact role played by these ingredients, versus the collateral constraints, in the amplification-persistence trade-off? To answer this question, this section studies a general-equilibrium version of KM's model with neo-classical capital and land accumulation. The interest of such a framework is that it is as close as possible to the standard business-cycle model: it relies on concave technologies, it endogenizes the interest rate and, finally, it abstracts from lumpy investment and introduces instead neo-classical input accumulation. The main lesson from this section is that with conventional input accumulation and convex preferences and technology, amplification and persistence turn out to go hand in hand under collateral constraints. Therefore, the amplification-persistence seems indeed specific to KM's setting and in particular to the assumption of lumpy investment.

The economy is composed of lenders and borrowers, both producing goods by using land, capital and fixed labor. Lenders provide one-period loans to borrowers. Credit can be used to finance consumption and investment. Lenders hold both land and capital and use output proceeds and interest income from previous loans to finance consumption and investment. The budget constraint of a representative lender is given by

$$
\tilde{C}_{t}+Q_{t}\left(\tilde{L}_{t+1}-\tilde{L}_{t}\right)+\tilde{K}_{t+1}-(1-\delta) \tilde{K}_{t}+B_{t+1}=\left(1+R_{t}\right) B_{t}+\tilde{Y}_{t}
$$


where $\tilde{C}_{t}$ is consumption, $\tilde{L}_{t}$ is the amount of land owned by the lender in the beginning of period $t, Q_{t}$ is the relative price of land in terms of the produced good, $\tilde{K}_{t+1}$ and $B_{t+1}$ are respectively the lender's capital stock and the amount of new loans generated in period $t, \tilde{Y}_{t}$ is lender's output, and $R_{t}$ is the real interest factor while $\delta \in(0,1)$ is the depreciation rate of capital. The lender maximizes the discounted value of utility streams

$$
\sum_{t=0}^{\infty} \tilde{\beta}^{t} \frac{\tilde{C}^{1-\tilde{\sigma}}}{1-\tilde{\sigma}},
$$

where the time discounting factor is $\tilde{\beta} \in(0,1)$ while $\tilde{\sigma} \geq 0$ measures relative risk aversion. The production technology available to the lender is given by

$$
\tilde{Y}_{t}=A \tilde{K}_{t}^{\tilde{\alpha}} L_{t}^{\tilde{\gamma}}
$$

where $\tilde{\alpha}, \tilde{\gamma} \in(0,1), \tilde{\alpha}+\tilde{\gamma}<1$, and $A$ is TFP.

Similarly, the representative borrower's production technology is given by

$$
Y_{t}=A K_{t}^{\alpha} L_{t}^{\gamma}
$$

where $\alpha, \gamma \in(0,1), \alpha+\gamma<1, L$ is the amount of land owned by the borrower, and $K$ denotes his capital stock. The total amount of land is in fixed supply, that is,

$$
L_{t}+\tilde{L}_{t}=\bar{L}
$$

where $\bar{L}$ is a positive number. A representative borrower in each period needs to finance consumption, land investment, capital investment, and loan payment. The budget constraint of the borrower is given by

$$
C_{t}+Q_{t}\left(L_{t+1}-L_{t}\right)+K_{t+1}-(1-\delta) K_{t}+\left(1+R_{t}\right) B_{t}=B_{t+1}+Y_{t}
$$


The representative borrower maximizes

$$
\sum_{t=0}^{\infty} \beta^{t} \frac{C_{t}^{1-\sigma}}{1-\sigma}
$$

with $\sigma \geq 0$. As in $\mathrm{KM}$, borrowers are assumed to be more impatient than lenders, that is, $\beta<\tilde{\beta}$.

The borrowing constraint faced by the borrower is, as in KM,

$$
\theta Q_{t+1} L_{t+1} \geq\left(1+R_{t+1}\right) B_{t+1}
$$

where $\theta \in(0,1]$ measures the collateral value of land. ${ }^{2}$ While KM assume $\theta=1$, I discuss below the consequences of setting $\theta<1$.

\section{Definition 3.1 (Competitive Equilibrium)}

An intertemporal competitive equilibrium with perfect foresight is a sequence of positive prices $\left\{Q_{t}, R_{t}\right\}_{t=0}^{\infty}$ and positive allocations $\left\{C_{t}, \tilde{C}_{t}, \tilde{K}_{t+1}, K_{t+1}, L_{t+1}, \tilde{L}_{t+1}\right\}_{t=0}^{\infty}$ such that:

(i) $\left\{C_{t}, \tilde{C}_{t}, \tilde{K}_{t+1}, K_{t+1}, L_{t+1}, \tilde{L}_{t+1}\right\}_{t=0}^{\infty}$ maximizes both (6) subject to (5) and (11) subject to (10)-(12), and satisfies the transversality conditions $\lim _{t \rightarrow \infty} \beta^{t} \Lambda_{t} L_{t+1}=0$, $\lim _{t \rightarrow \infty} \beta^{t} \Lambda_{t} K_{t+1}=0, \lim _{t \rightarrow \infty} \tilde{\beta}^{t} \tilde{\Lambda}_{t} \tilde{L}_{t+1}=0, \lim _{t \rightarrow \infty} \tilde{\beta}^{t} \tilde{\Lambda}_{t} \tilde{K}_{t}=0$, and the complementarity slackness condition, $\Phi_{t}\left[\theta Q_{t} L_{t}-\left(1+R_{t}\right) B_{t}\right]=0$ for all $t \geq 0$, given $\left\{Q_{t}, R_{t}\right\}_{t=0}^{\infty}$ and the initial endowments $L_{0} \geq 0, \tilde{L}_{0} \geq 0, B_{0} \geq 0, K_{0} \geq 0, \tilde{K}_{0} \geq 0$

\footnotetext{
${ }^{2}$ Introducing also non-depreciated capital as collateral turns out not to affect the main conclusion of this section.
} 
(ii) The good and asset markets clear for all $t \geq 0, C_{t}+\tilde{C}_{t}+K_{t+1}-(1-\delta) K_{t}+$ $\tilde{K}_{t+1}-(1-\delta) \tilde{K}_{t}=Y_{t}+\tilde{Y}_{t}$ and $L_{t}+\tilde{L}_{t}=\bar{L}$, respectively

The first-order conditions associated with condition $(i)$ of Definition 3.1 are stated in Appendix C. It is rather straightforward to show that the model has a unique stationary competitive equilibrium in which the borrower is credit-constrained so that condition (12) binds. So as to examine the quantitative implications of collateral constraints, the next step is to linearize the model around the steady state and to derive its impulse responses to a temporary, unexpected and serially-uncorrelated shock to TFP. More precisely, the following figures graph the responses of the linearized first-order conditions stated in Appendix $\mathrm{C}$ to a $\Delta \%$ deviation of $A$ from its steady state value. So as to parallel the discussion in Section 2 devoted to the KM model, the strategy is to assign numerical values to all parameters, except $\sigma$ and $\theta$ for which several levels are considered. More precisely, the idea is to show, by varying each parameter one by one, that the amplification-persistence does not necessarily hold.

In all cases presented below, the period is a quarter. I set the capital and land shares equal to $\gamma=\tilde{\gamma}=\alpha=\tilde{\alpha}=0.2$. In other words, capital and land are equally important in the production of goods while labor accounts for $60 \%$ of income. The rate of capital depreciation is set at $\delta=0.025$. The lender's rate of time discount equals $\tilde{\beta}=0.99$ (which implies an annual interest rate of $4 \%$ ), whereas the impatient borrower discounts future utility streams using $\beta=0.8 \tilde{\beta}$. Borrowers hold $10 \%$ of the land stock in steady state. Robustness analysis indicates that similar results 
hold when one slightly changes the above parameter values. As a benchmark, the lender's risk aversion is set to the conventional value $\tilde{\sigma}=1$, while the borrower's risk aversion is fixed at either $\sigma=1$ or $\sigma=10$. Such values belong to the range of parameter estimates obtained from micro-data (see e.g. Chiappori and Paiella [2]). On the other hand, the parameter controlling the tightness of the credit constraints takes two values: $\theta=1$ (in which case the full market value of the borrower's land stock is accepted as collateral by the lender) or $\theta=0.1$ (so that borrowing is limited to $10 \%$ of the land market value).

Two reasons account for my focusing on these two parameters. First, $\sigma$ determines the demand for credit emanating from the borrower-producer. In that sense, it plays a similar role to $\pi$ in the KM model studied in Section 2: the higher the borrower's relative risk aversion, the larger the increase in desired borrowing in the face of an unexpected TFP shock, for the usual insurance motive. Second, it is interesting to examine the impact of tightening the credit constraint, by lowering $\theta$. One thought experiment associated with such a decline in $\theta$ would be to try to figure out the impact of a contract clause that protects the borrowers by restricting the lender's claim to a fraction of - as opposed to the full - market value of the collateral in case of default. In other words, $\theta<1$ is interpreted as limited liability on the borrower's side. Alternatively, a lower $\theta$ could reflect some administrative costs that plague the imperfect recollection of the debt by the creditors. Cases (1a) and (1b): figures 1-2 depict the impulse responses of the borrower's debt, capital and land stocks and of land price, respectively when $\sigma=1$ - case (1a) - and $\sigma=10$ - case (1b) - while $\theta=1$ 
in both. These variables (except capital) also appear in the simulations provided by KM [7, p. 238] so that their figure 3 and the figures below may be directly compared. Contrasting figures 1 and 2 clearly reveals that amplification and persistence go and in hand. In other words, the trade-off featured in the KM economy does not occur. For example, the responses in period 2 of borrower's debt and land stocks increase respectively from $0.35 \%$ and $0.27 \%$ in figure 1 to $0.45 \%$ and $0.35 \%$ in figure 2. Similarly, the borrower's capital stock goes from a little less than $0.8 \%$ to $1.0 \%$. Unambiguously, increasing the borrower's relative risk aversion magnifies the effect of the TFP shock in the period following impact. On the other hand, persistence also is higher in figure 2 than in figure 1 . For instance, the half-life persistence ${ }^{3}$ of the TFP shock on borrower's debt is 6 quarters in figure 1 and 14 quarters in figure 2 , and it is higher for the other variables as well in figure 2. Notice that although the numbers in both figures seem small, they are not relative to what happens in a first-best environment, in which the impact of the TFP would vanish from period 2 thereafter (as shown by KM). In addition, the numbers in figures 1-2 are 2 order of magnitudes larger than the amplification levels reported in figure 3 of $\mathrm{KM}$ [7, p. 238]. The intuition of why there is no amplification-persistence trade-off here may be stated as follows. In contrast to the KM's economy, borrowers are risk-averse and use borrowing to smooth the impact of shocks on their consumption. Intuitively, one therefore expects that the larger their relative risk aversion $\sigma$, the larger amplification

\footnotetext{
${ }^{3}$ The half-life persistence of a shock indicates how long it takes for the initial impact of the shock to dissipate by half.
} 
of the impact of the TFP shock: following a $1 \%$ shock to TFP $A$ in period 1 , the responses of the borrower's debt stock, land stock, and land price in period 2 should be larger for larger $\sigma$ 's. This is confirmed in the numerical examples graphed in figures 1-2. In that sense, large $\sigma$ 's in my economy have the same effect on the amplification of the shock impact as large $\pi$ 's in KM's. However, what differs dramatically is the effect of both parameters on persistence: with large risk aversion, the mere desire of consumption smoothing also implies high persistence. After impact, the effects of the TFP shock tend to exhibit more persistence when risk aversion is larger because debt, land, and capital return more slowly to their steady-state levels. On the contrary, recall that such inertia of non-investing farmers arises only if the fraction of credit-constrained firms is small enough, which inevitably entails small amplification.

Cases (2a) and (2b): figures 3-4 graph the impulse responses respectively when $\sigma=1$ - case (2a) - and $\sigma=10$ - case $(2 \mathrm{~b})$ - while $\theta=0.1$ in both. Comparing both figures confirms again the absence of a trade-off between amplification and persistence of the impact of the TFP shock in period 1 . When only $10 \%$ of the market value of land is accepted as collateral by the lender, amplification and persistence still move together for the very same reason of consumption smoothing. Note that the land price is more volatile when $\theta=0.1$ : with tighter credit constraints, fluctuations in the price of collateral tend to be more pronounced. 
Fig.1: Impulse Responses to a TFP shock in Case (1a)

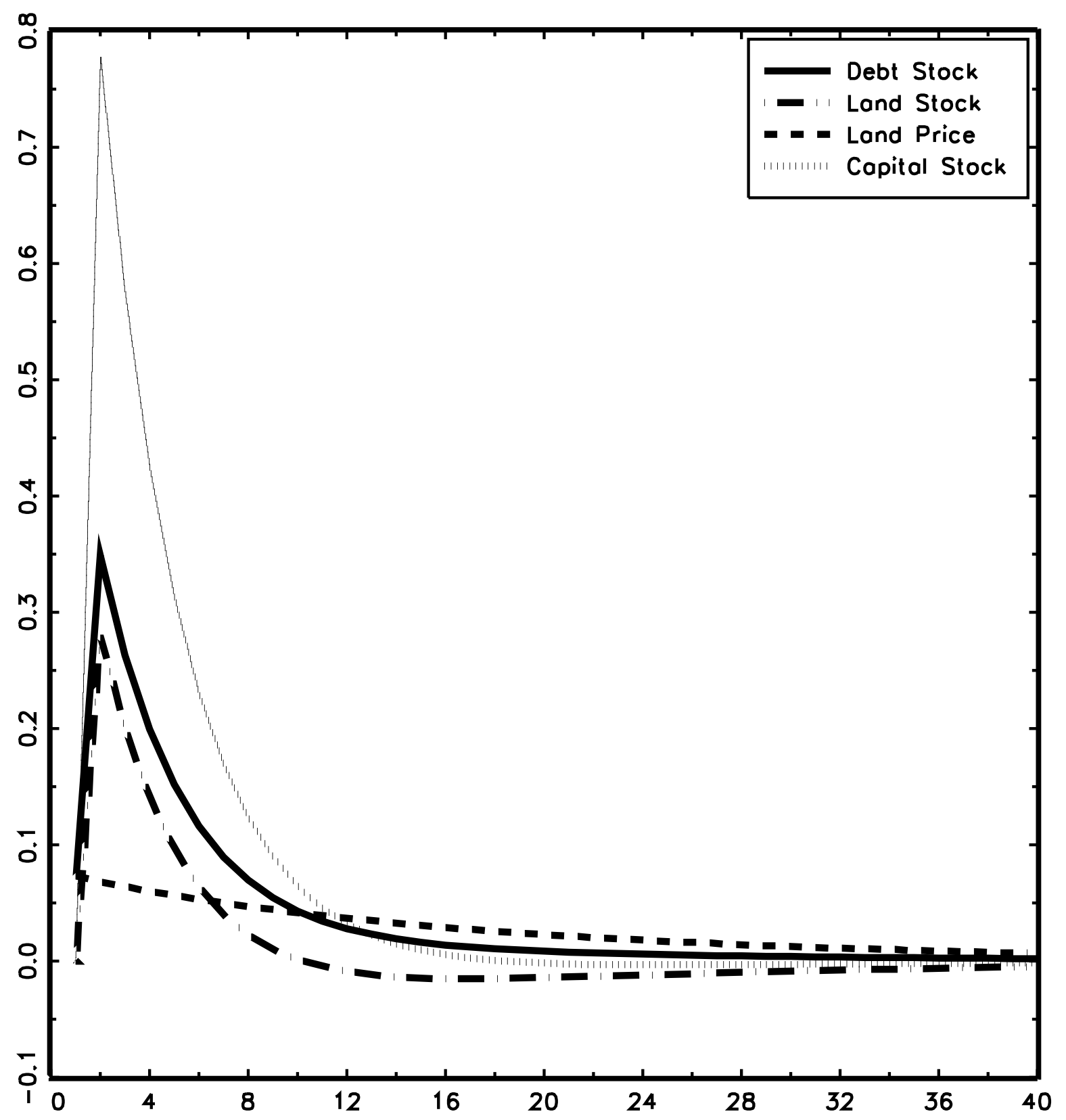


Fig.2: Impulse Responses to a TFP shock in Case (1b)

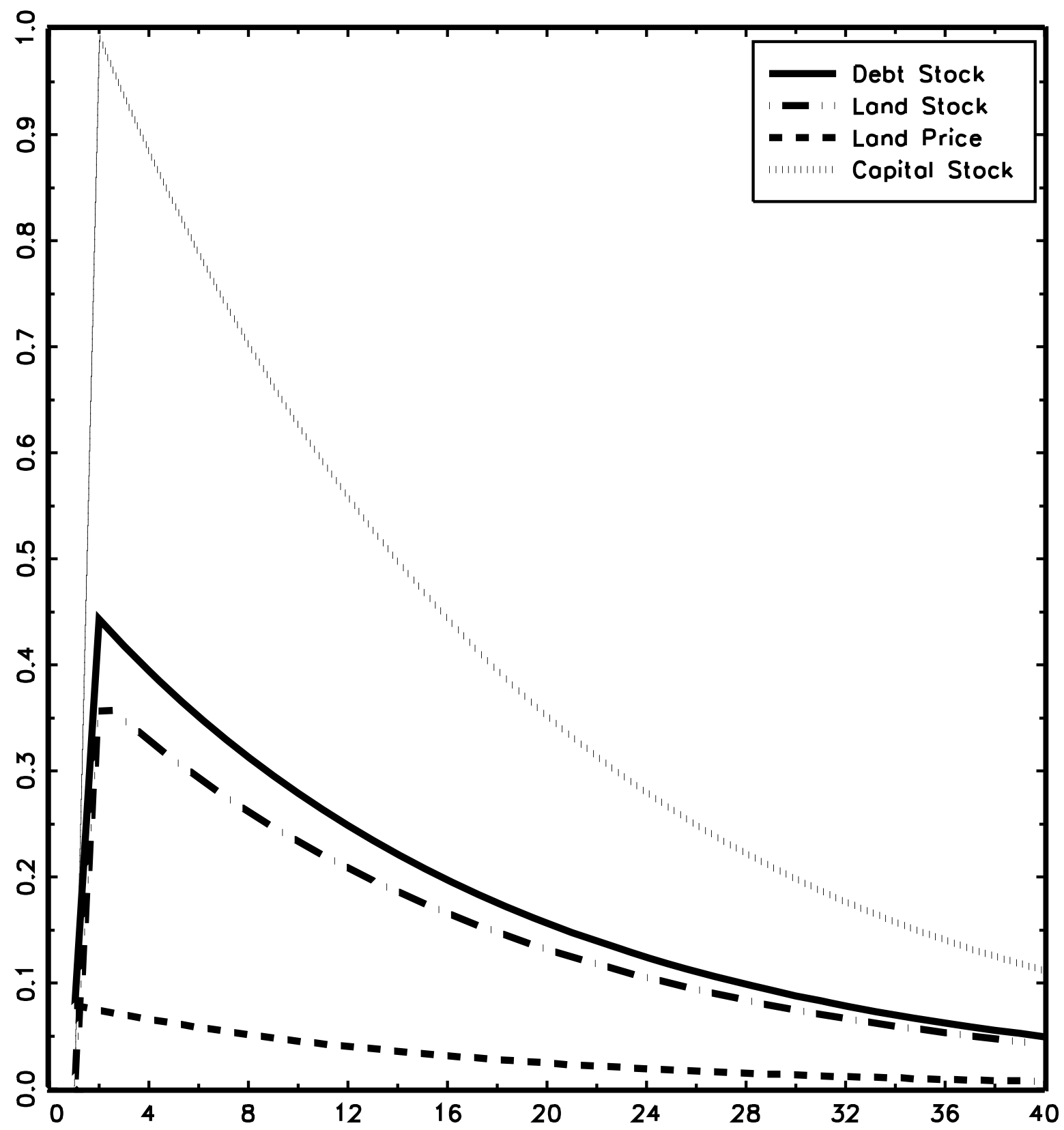


Fig.3: Impulse Responses to a TFP shock in Case (2a)

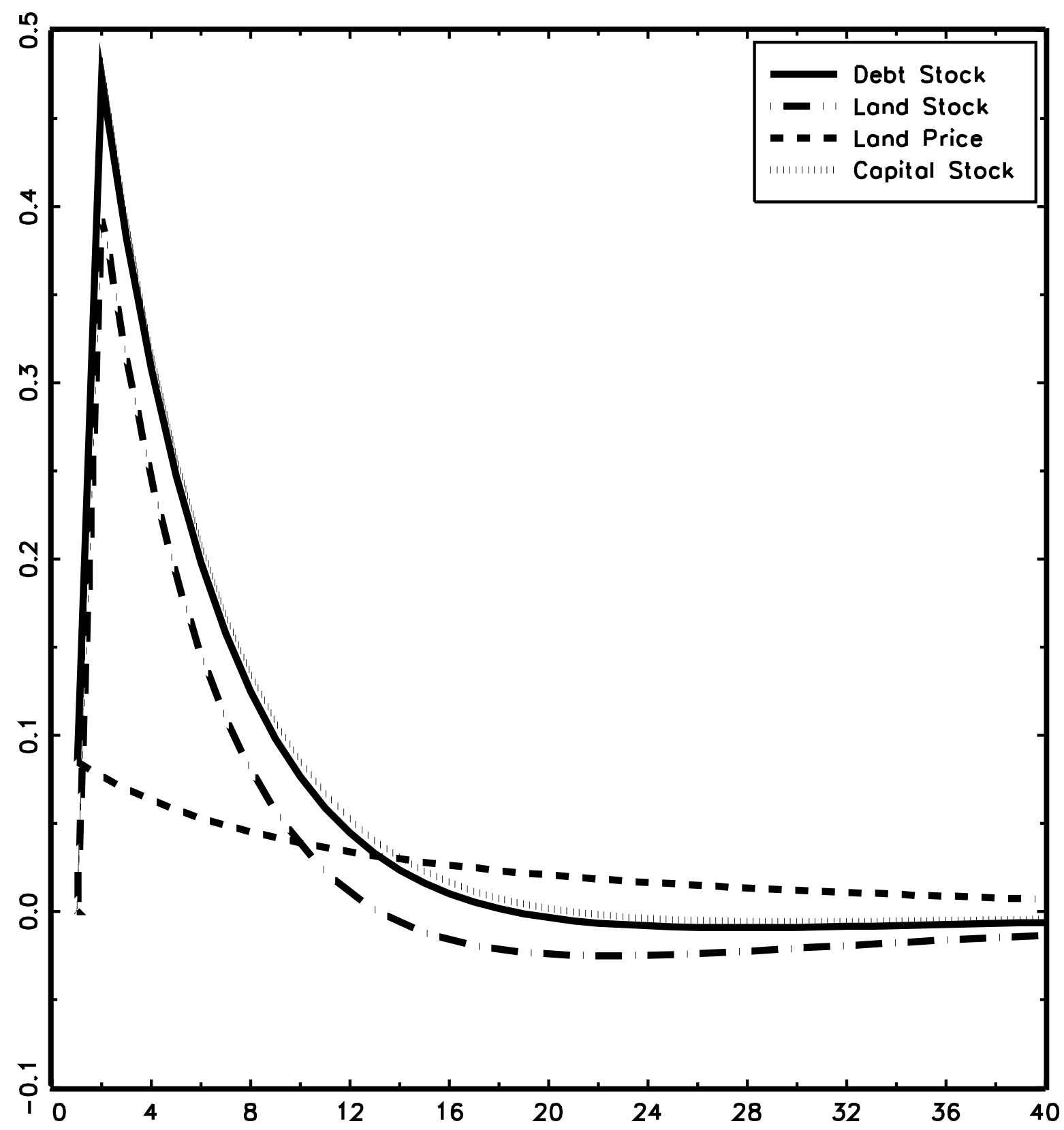


Fig.4: Impulse Responses to a TFP shock in Case (2b)

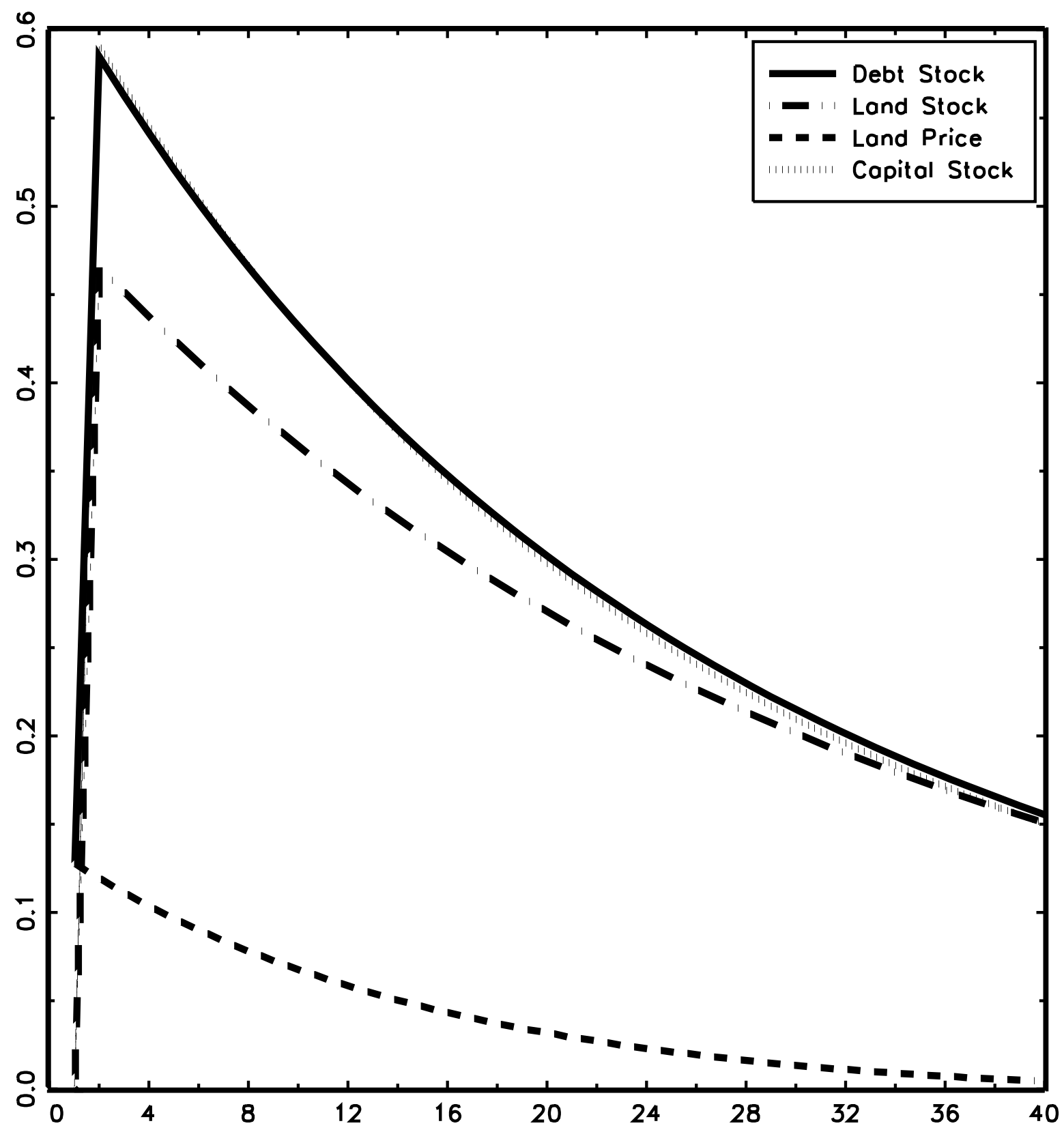




\section{Conclusion}

I have restated and discussed the amplification-persistence trade-off that arises in the model of Kiyotaki and Moore [7]. The key point behind this trade-off is that persistence of temporary productivity shocks at impact requires the fraction of creditconstrained firms to be small enough, so that a low fraction of farmers invest in each period. However, large amplification relies on just the opposite condition, as it is substantial only when there are many credit-constrained firms. The main result of this paper is to show by way of an example that such a trade-off does not extend to a general-equilibrium two-agent model that dispenses with several unorthodox assumptions made by Kiyotaki and Moore [7]. In particular, the benchmark model, featuring concave utility and production functions and neo-classical input accumulation, does not predict an amplification-persistence trade-off to occur because of collateral constraints. Although the model is arguably standard in many dimensions (it is indeed similar to Cordoba and Ripoll's [3]), it remains to be seen if the same conclusion stands in alternative settings with different credit market frictions.

Although this paper confirms some existing results and conjectures, it also calls for further research. The main lesson learned from the contribution by Cordoba and Ripoll [3] remains valid here: when capital accumulation and fractional collateral are added to the analysis, collateral constraints still have small quantitative effects, especially on aggregate output. ${ }^{4}$ However, this fact does not imply that collateral

\footnotetext{
${ }^{4}$ It is expected that introducing elastic labor would improve output amplification.
} 
constraints are quantitatively insignificant. As a matter of fact, this conclusion reinforces the importance of a major conjecture of Kiyotaki and Moore [7]: collateral constraints may have significant effects when interacted with other economic mechanisms, such as lumpy investment. Along that line, Pintus and Wen [11] study a model in which the interplay of collateral constraints and habit formation lead to credit cycles but rule out the amplification-persistence trade-off. In view of this example, it seems worthwhile to extend the research program pointed out by Kocherlakota [8] by acknowledging that a major challenge is to identify which interactions involving credit constraints, if any, have a prominent role in business-cycle theory.

\section{A Proof of Proposition 2.1}

From Kiyotaki and Moore [6, p. 33], one gets the expression of the characteristic polynomial associated with the Jacobian of eqs. (1), that is, $P(x)=(x-R) p(x)$, where $p(x)=x^{2}-T x+D$ and, under the additional requirement that $\phi=0$ :

$$
\begin{aligned}
D & =\eta \lambda R(1-\pi) /(\eta+1-\lambda+\lambda \pi), \\
T & =D+[\eta+R(1-\pi)(1-\lambda)] /(\eta+1-\lambda+\lambda \pi) .
\end{aligned}
$$

Therefore, $R>1$ is an unstable eigenvalue, while the other two are stable under the maintained assumptions, as I now show. A straightforward way to proceed is 
analyzing how $T$ and $D$ vary when $\pi$ is decreased from one. That is, define:

$$
\begin{aligned}
D(\pi) & \equiv \eta \lambda R(1-\pi) /(\eta+1-\lambda+\lambda \pi)>0, \\
T(\pi) & \equiv D(\pi)+[\eta+R(1-\pi)(1-\lambda)] /(\eta+1-\lambda+\lambda \pi)>0,
\end{aligned}
$$

where $\eta>0$. One easily gets that $D(\pi)$ is a strictly decreasing function of $\pi$, with $D(1)=0$. On the other hand, $0<T(\pi)<1+D(\pi)$ for all $\pi$ in the range allowed by Assumption 2.1. Therefore, local saddle-point stability is ensured provided that $D(0)<1$. Several cases have to be considered.

(i) If $\lambda R<1$, then it is easy to show that $D(0)=\eta \lambda R /(\eta+1-\lambda)<1$. In that case, $p(x)$ has two stable roots and the steady state is a saddle under Assumption 2.1 .

(ii) If $\lambda R>1$, then two subcases arise, depending on whether $\eta$ is smaller or larger than $(1-\lambda) /(\lambda R-1)$. If $\eta<(1-\lambda) /(\lambda R-1)$, then $D(0)<1$. If $\eta>(1-\lambda) /(\lambda R-1)$, then there exists a critical value $\pi_{H} \equiv(\lambda(\eta R+1)-1-\eta) /[\lambda(1+\eta R)]$ such that $D\left(\pi_{H}\right) \equiv 1$ and $D(0)<1$ if and only if $\pi>\pi_{H}$. It turns out that $\pi_{H}<1-1 / R$ so that Assumption 2.1 rules out $D(0)>1$. This proves that in both cases $(i)$ and $(i i)$, $p(x)$ has two stable roots and the steady state is a saddle under Assumption 2.1. 


\section{B Proof of Theorem 2.1}

From the expressions in eqs. (4), one gets that the decay rate $\mu \equiv 1-\sqrt{D}$ is an increasing function of $\pi$, as $\sqrt{D}$, or to put it differently, persistence, is a decreasing function of $\pi$. In particular, the decay rate increases from zero to one when $\pi$ goes up from $\pi_{H}$ to one. I now show that amplification of capital and output increases with $\pi$. In Proposition 2.1, the steady state is a saddle that possesses one unstable eigenvalue (that is, $R>1$ ) and two eigenvalues lying inside the unit circle (that are complex conjugate provided that $\pi$ is not too close to one). Therefore, the requirement that $\lim _{s \rightarrow \infty} E_{t}\left(R^{-s} q_{t+s}\right)=0$ for all $t \geq 1$ ruling out exploding bubbles implies that the dynamics are restricted to the stable manifold of the saddle. Denote by $\Delta$ the (small) unexpected, one-period productivity shock, measured in percentage terms, that hits the economy while it is at steady state. On the other hand, denote by hatted variables, the percentage deviation from steady state. For example, $\hat{q} \equiv\left(q-q^{*}\right) / q^{*}$ denotes the current deviation of $q$, the land price, from its steady state value $q^{*}$. Then linearizing eqs. (1) and restricting the analysis to the (linear) two-dimensional, stable manifold allows one to derive the following expressions, when $\lambda=1$ :

$$
\hat{q} / \Delta=1 / \eta, \hat{L} / \Delta=\pi[\eta+R /(R-1)] /(\eta+\pi)
$$

Moreover, output aggregated over farmers and non-farmers is given by $Y=(a+$ c) $L+G(\bar{L}-L)$. At steady state, one has $Y^{*}=(a+c-R a) L^{*}+R a \bar{L}$. Therefore, one has that $\hat{Y}=\hat{L}(a+c-R a) /\left(a+c-R a+R a \bar{L} / L^{*}\right)<\hat{L}$. Collecting all facts, 
with $\lambda=1$, one gets that:

$$
\hat{Y} / \Delta=\pi(a+c-R a)[\eta+R /(R-1)] /\left[(\eta+\pi)\left(a+c-R a+R a \bar{L} / L^{*}\right)\right] .
$$

Now it is straightforward to show, by using eqs. (15) that while $\hat{q} / \Delta$ is independent of $\pi$, one has that $d(\hat{L} / \Delta) / d \pi=\eta[\eta+R /(R-1)] /(\eta+\pi)^{2}>0$. In other words, capital amplification is an increasing function of $\pi$. Finally, noting that both $\bar{L}$ (by assumption) and $L^{*}$ are independent of $\pi$, one concludes from eq. (16) and the above finding that output amplification $\hat{Y} / \Delta$ is also an increasing function of $\pi$. This completes the proof of the statement that there exists an amplificationpersistence trade-off, as persistence (resp. amplification) decreases (resp. increases) when the share of credit-constrained farmers $\pi$ goes up from $\pi_{H}$ to one. By continuity, the amplification-persistence trade-off remains valid when $\lambda$ is close enough to one, which is bound to be the only plausible configuration if the period is short (say, a quarter).

\section{Definition 3.1: First-Order Conditions}

Denoting $\tilde{\Lambda}$ as the Lagrangian multiplier of the constraint (5), the first-order conditions of the lender with respect to consumption, land, capital, and lending are given, respectively, by

$$
\tilde{C}_{t}^{-\tilde{\sigma}}=\tilde{\Lambda}_{t}
$$




$$
\begin{gathered}
Q_{t} \tilde{\Lambda}_{t}=\tilde{\beta} Q_{t+1} \tilde{\Lambda}_{t+1}+\tilde{\beta} \tilde{\gamma} \tilde{\Lambda}_{t+1} \tilde{Y}_{t+1} / \tilde{L}_{t+1} \\
\tilde{\Lambda}_{t}=\tilde{\beta} \tilde{\Lambda}_{t+1}\left(\tilde{\alpha} \tilde{Y}_{t+1} / \tilde{K}_{t+1}+1-\delta\right) \\
\tilde{\Lambda}_{t}=\tilde{\beta}\left(1+R_{t+1}\right) \tilde{\Lambda}_{t+1}
\end{gathered}
$$

Denoting $\{\Lambda, \Phi\}$ as the Lagrangian multipliers of constraints (10) and (12), respectively, the first-order conditions of the borrower with respect to consumption, land, capital, and borrowing are given, respectively, by

$$
\begin{gathered}
C_{t}^{-\sigma}=\Lambda_{t} \\
Q_{t} \Lambda_{t}=\beta Q_{t+1} \Lambda_{t+1}+\beta \gamma \Lambda_{t+1} Y_{t+1} / L_{t+1}+\theta Q_{t+1} \Phi_{t} \\
\Lambda_{t}=\beta \Lambda_{t+1}\left(\alpha Y_{t+1} / K_{t+1}+1-\delta\right) \\
\Lambda_{t}=\beta\left(1+R_{t+1}\right) \Lambda_{t+1}+\left(1+R_{t+1}\right) \Phi_{t}
\end{gathered}
$$

\section{References}

[1] Aghion, P., Banerjee, A., Piketty, T., 1999. Dualism and macroeconomic volatility. Quarterly Journal of Economics 114, 1359-97.

[2] Chiappori, P.-A., Paiella, M., 2008. Relative risk aversion is constant: evidence from panel data. Discussion papers 5-2008, University of Naples "Parthenope".

[3] Cordoba, J.-C., Ripoll, M., 2004. Credit cycles redux. International Economic Review 45, 101146.

[4] Freixas, X., Rochet, J.-C., 1997. Microeconomics of banking. MIT Press, Cambridge.

[5] Kiyotaki, N., 1998. Credit and business cycles. Japanese Economic Review 49, 18-35. 
[6] Kiyotaki, N., Moore, J., 1995. Credit cycles. NBER working paper series n. 5083.

[7] Kiyotaki, N., Moore, J., 1997. Credit cycles. Journal of Political Economy 105, 211-48.

[8] Kocherlakota, N., 2000. Creating business cycles through credit constraints. Federal Reserve Bank of Minneapolis Quarterly Review 24, 2-10.

[9] Matsuyama, K., 2007. Credit traps and credit cycles. American Economic Review 97, 503-16.

[10] Pintus, P., 2007. Credit market frictions and the amplification-persistence trade-off. GREQAM working paper 2007-26.

[11] Pintus, P., Wen, Y., 2008. Excessive demand and boom-bust cycles. Federal Reserve Bank of St. Louis working paper 2008-014. 